\title{
Laryngeal Obstruction, CTCAE
}

National Cancer Institute

\section{Source}

National Cancer Institute. Laryngeal Obstruction, CT CAE. NCI Thesaurus. Code C143639.

A disorder characterized by blockage of the laryngeal airway. 